\title{
A 4D-Trajectory Planning Method Based on Hybrid Optimization Strategy for Demand and Capacity Balancing
}

\author{
Yutong Chen ${ }^{a, b}$, Yan $\mathrm{Xu}^{a}$, Minghua $\mathrm{Hu}^{b}$, Fei Huang ${ }^{b}$, Qi Nie ${ }^{b}$ \\ ${ }^{a}$ Cranfield University \\ Bedford, United Kingdom \\ \{yutong.chen, yanxu\}@cranfield.ac.uk \\ ${ }^{b}$ Nanjing University of Aeronautics and Astronautics \\ Nanjing, China \\ \{chenyutong, minghuahu, hfei_11, heynieqi\}@nuaa.edu.cn
}

\begin{abstract}
To effectively solve the Demand and Capacity Balancing (DCB) in future Trajectory-Based Operation (TBO) scenarios, this article first proposes a pre-tactical-and-tactical integrated Four-Dimensional Trajectory (4DT) planning framework. The framework decomposes large-scale 4DT planning into two stages, namely, the General 4DT (G4DT) planning in the pre-tactical stage and the Special 4DT (S4DT) planning in the tactical stage. A Hybrid Optimization Strategy (HOS) based planning method is designed for G4DT planning. In this method, the sequential decision architecture based on time window, heuristic strategy (greedy strategy) and optimization algorithm are combined to realize the fast trajectory planning of large-scale flights. In the optimization model based on continuous time, the nonlinear model is transformed into a linear model by constructing the flight conflict correlation matrix, which greatly improves the solving speed of the model. Real flight schedule data for French and Spanish airspace were used to verify the effectiveness and efficiency of the HOS method. This method is compared with Computer-Assisted Slot Allocation (CASA). The results show that the proposed method can effectively reduce the flight delay time and improve the flight on-time rate. Due to its fast operation speed, the proposed method has great potential to dynamically update the planning results according to the realtime air space operation status in actual operation.
\end{abstract}

Keywords-trajectory-based operation; demand and capacity balancing; hybrid optimization strategy; time window; sequential decision

\section{INTRODUCTION}

At present, the main contradiction in the development of civil aviation in the world is the contradiction between the increasing total volume of transportation and the increasingly saturated airspace capacity, that is, the contradiction between demand and capacity. Therefore, how to balance the demand and capacity has gradually become a concern of the field. In 2005, ICAO listed Demand and Capacity Balancing (DCB) as one of the seven modules of the concept of Air Traffic Management (ATM) operation. It is pointed out that the management of demand and capacity imbalance is mainly based on the predictability of the system and the system needs to be able to cope with unexpected events [1]. Thus, the implementation effect of DCB is mainly affected by uncertainty, which mainly includes two aspects: one is the uncertainty of flight and the other is the uncertainty of airspace. The uncertainty of flight mainly comes from the uncertainty of conflict, control, pilot's operation, and air situation. On the one hand, the uncertainty of airspace comes from dangerous weather and military aviation activities, and on the other hand, it comes from the change of airspace performance caused by the uncertainty of flight operation. Therefore, how to deal with the uncertainty in operation becomes one of the key issues in the effective implementation of DCB.

At present, air traffic is mainly based on air route operation, which takes the form of traffic flow. Therefore, the current DCB is mainly carried out in the form of Air Traffic Flow Management (ATFM) or Air Traffic Flow and Capacity Management (ATFCM) [2]. Its main operation means include Ground Delay Program (GDP), rerouting, and their combination. Computer-assisted Slot Allocation (CASA) is a commonly used DCB method in practice at present. It can balance demand and capacity by identifying the sector with capacity overload and adopting GDP for the flights passing through it. There are two main problems with CASA: one is that it tends to result in high delays; On the other hand, it only considers that the moment when aircraft enters the sector in the hotspot meets the minimum time interval requirement, while flights in the non-hotspot do not need to be affected by this limit. Therefore, in the non-hotspot, multiple flights may enter the sector in a short period [3]. To better balance demand and capacity, a DCB model for air traffic network optimization is proposed. This method is based on the pseudo-EulerianLagrange flow model, which can get a single ATFM measure close to the optimal in a very short time, but seems to lack the potential for large-scale problems [4]. With the development and application of artificial intelligence, a DCB technology based on reinforcement learning implemented in the pretactical stage is proposed, aiming to learn how to solve the DCB problem by using GDP through artificial intelligence. However, the sector model considered by this method is too simple and the uncertainty factors are not considered [5]. Although AFTM promotes the orderly operation of flights and improves the utilization rate of airspace resources to a certain extent, it still has great uncertainty in operation due to its relatively rough restrictions in the implementation process [6]. 
Fortunately, thanks to the rapid development of Communication, Navigation and Surveillance (CNS) equipment and technology, the concept of Trajectory Based Operation (TBO) has emerged and been gradually applied in practice. The trajectory describes the position characteristics of aircraft in the horizontal dimension, vertical dimension, and time dimension, where Four-Dimensional Trajectory (4DT) refers to the determination of aircraft trajectory by combining traditional three-dimensional spatial information (longitude, latitude, and altitude) with temporal information. Considering flight parameters such as weight, altitude and speed, accurate and continuous trajectory information can be obtained. TBO takes the flight trajectory information of the aircraft as the only reference and shares the flight trajectory in the ATM system. All relevant parties make decisions together to accurately manage and control the operation of the aircraft. The essence of TBO is to use the flight trajectory information as the medium to realize the fine collaborative decision-making covering the entire ATC system, to achieve the purpose of safety and efficiency [7]. The existing air traffic management decision support system based on 4DT can realize multi-objective trajectory optimization through autonomous 4DT planning, negotiation, and verification [8]. The ATFCM technology supported by TBO technology can transform the original nonlinear programming problem into a linear programming problem, and the goal of resolving potential conflicts can be achieved only by making small changes to the flight speed, which will effectively reduce the complexity of the problem and the uncertainty of operation [9]. To take advantage of TBO technology to better solve the problem of DCB, a pre-tactical ATFM optimization based on mixed-integer linear programming model is proven to reduce $97 \%$ of the delay. Due to the application of linear holding technology, its cost of reducing delay is just an average extra consumes less than 100 kilogram of fuel and extra 50 euro of route charge [10]. Therefore, the application of $\mathrm{TBO}$ can greatly reduce the uncertainty of flight operation, and DCB based on flight trajectory management will be one of the key technologies in the current and future development of ATM.

However, in terms of the uncertainty of airspace, although some prediction techniques based on airspace have been proposed, the overall prediction accuracy has not reached the ideal level. This is determined by the characteristics of its influencing factors. For example, military aviation activities are usually highly confidential and can only be known by civil aviation until near implementation. Weather conditions are also fickle. Although the level of weather forecasting technology continues to improve, it is still not accurate enough. Special situation is even more unpredictable. Therefore, to deal with the uncertainty in the airspace that is difficult to accurately predict, a fast 4DT planning technology for large-scale DCB problems is urgently needed to dynamically update the planning results according to the real-time operation conditions, to achieve efficient DCB.

Based on the above reasons, this article carries out the following work:

1. A set of the pre-tactics-and-tactical integrated 4DT planning framework for TBO operation scenarios is proposed. The large-scale non-conflict 4DT planning is decomposed into two stages, namely pre-tactical and tactical 4DT planning, which are progressively and interlinked. The concepts of General 4DT (G4DT) and Special 4DT (S4DT) are defined.

2. The difficulties of G4DT planning for DCB under TBO operation scenarios are analyzed, and a set of G4DT planning method based on Hybrid Optimization Strategy (HOS) is designed, which combines heuristic framework with the exact solution, that is, integrates the idea of sequential decision, greedy strategy and optimal solution based on time window.

3. The performance of the proposed method is tested through real data and compared with the CASA used in actual operation. The experimental results prove the effectiveness and high efficiency of the proposed method.

\section{A PRE-TACTICS-AND-TACTICAL INTEGRATED 4DT PLANNING FRAMEWORK}

In the TBO scenario, each flight will be assigned a conflictfree 4DT to ensure that when all flights strictly follow the flight trajectory, there will be no conflict during operation. Largescale 4DT planning is extremely difficult and time-consuming (e.g., 4DT for thousands to tens of thousands of flights across a country), so phased planning is an easy solution to come up with. Therefore, this article proposes a phased implementation of pre-tactical trajectory planning and tactical trajectory planning and forms a dynamically updated pre-tactics-andtactical integrated 4DT planning framework (as shown in Figure 1).

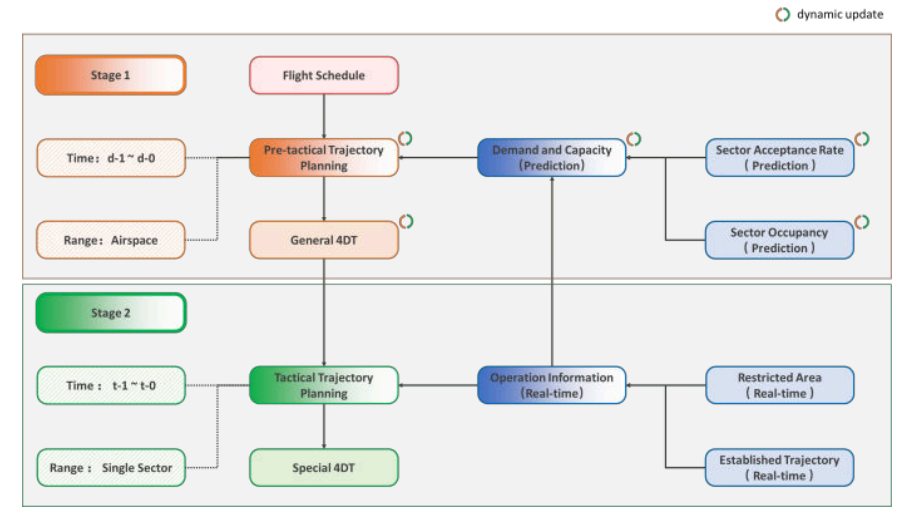

Figure 1. The pre-tactical-and-tactical integrated 4DT planning framework.

\section{Definitions:}

General 4DT (G4DT): a 4DT composed of fourdimensional information (three-dimensional coordinates and their Control Time of Arrival (CTA)) about the entry and exit points of all sectors of the flight planning trajectory. Wherein, the exit point of the previous sector of the trajectory is the entry point of the next sector.

Special 4DT (S4DT): a continuous 4DT calculated from the flight trajectory, the CTA by the starting and ending points, and the aircraft's dynamic performance parameters. The starting and ending points are the entry points and exit points of the flight within a certain sector. 
Sector Acceptance Rate: the number of flights accepted per sector per unit of time, generally expressed as the reciprocal of sector capacity per unit of time. If the capacity of a certain sector is 40 flights in 20 minutes, then the acceptance rate of this sector is $40 / 20=2$ flights per minute, then in the TBO operation, the minimum entry time interval of any two flights entering this sector should be greater than 0.5 minutes.

Sector Occupancy: the entry time of a flight for which this sector has been confirmed to be occupied. These confirmed entry times are from flights that have already taken off, as they have completed final pre-tactical trajectory planning.

Restricted Area: an area in a sector that is inaccessible to flights due to military activities, hazardous weather, etc.

Established Trajectory: the agreed trajectory of flights operating in the sector or of flights about to enter the sector for which the agreed trajectory has been in effect.

Flight Schedule: including the flight's departure and arrival at the airport and time, route sectors, three-dimensional coordinates of entry points and exit points of corresponding sectors, and the crossing time of these points calculated according to the flight performance of the flying model, the G4DT of the flight can be calculated through the flight schedule.

General Conflict-free: refers to the absence of conflict between G4DT, that is, the entry time of any two flights passing through the same sector in this sector meets the sector acceptance limit.

Special Conflict-free: refers to the absence of conflict between S4DT, that is, two flights meet the minimum safety separation requirements at any time, including horizontal separation and vertical separation.

Stage 1 is the pre-tactical trajectory planning stage, d- 1 to $\mathrm{d}-0$, that is, the day before the flight operation to the operation day, the operation unit is a range of airspace, such as one or more national airspace, one or more flight information areas, several freely combined sectors, etc. Based on the flight schedule in the current operation unit and the predicted demand and capacity of each sector, the G4DT of all flights is obtained through centralized optimization. Sector demand and capacity include sector acceptance rate (capacity) and sector occupancy (demand). The demand and capacity prediction of the sector is based on the real-time sector operation, so the prediction results are dynamically updated with time, and the prediction accuracy increases gradually as the time approaches the takeoff time. Therefore, to obtain a more accurate and effective G4DT before the flight takes off, the pre-tactical trajectory planning will be an iterative updating process, and the G4DT obtained will also be updated dynamically.

Stage 2 is the tactical trajectory planning stage, and the operation time is from $\mathrm{t}-1$ to $\mathrm{t}-\mathrm{0}$, that is, the time from one minute before the flight enters the target sector to the time when it just enters the sector. The operation unit is a single sector, that is, the sector that is about to enter at present. Based on the entry point and exit point of the G4DT of the current flight in the sector, and the operation of the sector, the S4DT of the flight is optimized. Among them, the real-time sector operation situation includes restricted area and established trajectory. Once the S4DT is determined, the flight will be strictly implemented and will not be changed without special circumstances.

The above two stages together constitute the pre-tacticaland-tactical integrated 4DT planning framework to realize the generation of conflict-free 4DT during TBO operation. Tactical phase trajectory planning techniques have been studied before, so we will not discuss them in this article. Those who are interested may refer to our previously published papers [11]. In this article, we mainly discuss the G4DT planning method in the pre-tactical stage (stage 1). Besides, we only consider solving the DCB problem through GDP. On the one hand, this is to simplify the problem. On the other hand, GDP has a better performance in reducing the system uncertainty than rerouting $[12]$.

\section{A G4DT PLANNING METHOD BASED ON HYBRID OPTIMIZATION STRATEGY}

\section{A. Problem Description}

The problem to be solved in this article is as follows: for large-scale flight operation scenarios, in the pre-tactical stage, the system obtains the take-off time of each flight and the time of entering each sector (that is, G4DT) according to the flight schedule. By imposing a delay time on some of the flights (that is, GDP), the system enables all flights to improve the on-time rate as far as possible under the precondition of satisfying the general conflict-free constraint, that is, the number of delayed flights is as low as possible.

\section{B. Assumptions}

a) The flight time in a particular sector is constant.

b) The departure time of the flight can only be delayed, not advanced.

c) Only when the flight is operating in the en-route airspace is considered.

\section{Problem Analysis}

For large-scale pre-tactical 4DT planning problem (such as the thousands of flights a day and hundreds of sectors), if the constraint programming optimization solution, taking minimum delay flight number as the optimization goal, whether using the mixed-integer nonlinear programming model based on continuous-time or the integer nonlinear programming model based on the discrete-time model, none of the optimal results can be solved in limited time. A relatively simple integer nonlinear programming model is taken as an example to illustrate the scale of the problem. In the actual optimization modelling, the integer programming model is usually constructed into a $0-1$ integer programming model to make the model easier to solve. The objective function can be expressed as

$$
\min Z=\sum_{i \in I} x_{i}
$$


where $x_{i}$ is the $0-1$ decision variable. 1 and 0 represent the flight $i$ is delayed or not, respectively. The constraints can be expressed as

$$
\begin{gathered}
\sum_{j \in\left\{0,1, \ldots, N_{i}\right\}} d_{i j}=x_{i}, i \in I \\
\left|\left(\sum_{j \in\left\{0,1, \ldots, N_{i}\right\}} x_{i j} j T+E_{i k}\right)-\left(\sum_{j^{\prime} \in\left\{0,1, \ldots, N_{i^{\prime}}\right\}} x_{i^{\prime} j^{\prime}} j T+E_{i^{\prime} k}\right)\right| \geq \frac{1}{a_{k}}, \\
k \in K, i, i^{\prime} \in I_{k}, i \neq i^{\prime} \\
x_{i} \in\{0,1\}, i \in I \\
d_{i j} \in\{0,1\}, i \in I, j \in\left\{0,1, \ldots, N_{i}\right\}
\end{gathered}
$$

In Formula (2), $d_{i j}$ is the $0-1$ decision variable. 1 and 0 represent the flight $i$ is delayed for $j$ unit time or not. $N_{i}$ is a constant that represents the maximum unit time that the flight $i$ can be delayed. Therefore, Formula (2) means that when $x_{i}$ is 0 , the right side of the equation is 0 , so all $d_{i j}$ on the left side of the equation can only be 0 , that is, no flight delay occurs; When $x_{i}$ is 1 , the right side of the equation is 1 , so there is a unique $d_{i j}$ on the left side of the equation is 1 , that is, the flight $i$ is delayed for a certain unit time. Since the objective function of the model is to minimize the sum of $x_{i}$, Formula (2) can be rewritten as

$$
\sum_{j \in\left\{0,1, \ldots, N_{i}\right\}} d_{i j} \leq x_{i}, i \in I
$$

In Formula (3), $E_{i k}$ is a constant, determined by the flight plan, and represents the time when flight $i$ enters sector $k$ in the flight plan. $T$ is a constant which represents a unit time. $K$ is the set of all sectors and $I_{k}$ is the set of all flights passing through sector $k . a_{k}$ is a constant, representing the acceptance rate of sector $k$, then $1 / a_{k}$ is the minimum entry time interval of any two flights entering the same sector. Since there is absolute value calculation on the left side of the inequality in Formula (3), according to the requirements of the constrained programming model, Formula (3) should be rewritten as

$$
\begin{aligned}
& \left(\begin{array}{l}
\left.\sum_{j \in\left\{0,1, \ldots, N_{i}\right\}} x_{i j} j T+E_{i k}\right)- \\
\left(\sum_{j^{\prime} \in\left\{0,1, \ldots, N_{i^{\prime}}\right\}} x_{i^{\prime} j^{\prime}} j T+E_{i^{\prime} k}\right)-\frac{1}{a_{k}}
\end{array}\right) \delta_{i i^{\prime} k}^{+} \geq 0, k \in K, i, i^{\prime} \in I_{k}, i \neq i^{\prime} \\
& \left(\begin{array}{c}
\left.\sum_{j \in\left\{0,1, \ldots, N_{i}\right\}} x_{i j} j T+E_{i k}\right)- \\
\left(\sum_{j^{\prime} \in\left\{0,1, \ldots, N_{i^{\prime}}\right\}} x_{i^{\prime} j^{\prime}} j T+E_{i^{\prime} k}\right)+\frac{1}{a_{k}}
\end{array}\right) \delta_{i i^{\prime} k}^{-} \leq 0, k \in K, i, i^{\prime} \in I_{k}, i \neq i^{\prime}
\end{aligned}
$$

$$
\begin{gathered}
\delta_{i i^{\prime} k}^{+}+\delta_{i i^{\prime} k}^{-} \geq 1, k \in K, i, i^{\prime} \in I_{k}, i \neq i^{\prime} \\
\delta_{i i^{\prime} k}^{+}, \delta_{i i^{\prime} k}^{-} \in\{0,1\}, k \in K, i, i^{\prime} \in I_{k}, i \neq i^{\prime}
\end{gathered}
$$

where $\delta_{i i^{\prime} k}^{+}$and $\delta_{i i^{\prime} k}^{-}$are $0-1$ auxiliary variables used to transform the absolute value expression in Formula (3) to make it conform to the requirements of the constrained optimization model.

In summary, the 0-1 integer programming nonlinear programming model can be summarized as

$$
\begin{aligned}
& \min Z=\sum_{i \in I} x_{i} \\
& \text { s.t. } \\
& \left\{\begin{array}{l}
\sum_{j \in\left\{0,1, \ldots, N_{i}\right\}} d_{i j} \leq x_{i}, i \in I \\
\left(\begin{array}{l}
\left.\sum_{j \in\left\{0,1, \ldots, N_{i}\right\}} x_{i j} j T+E_{i k}\right) \\
\left(\begin{array}{l}
\sum_{j^{\prime} \in\left\{0,1, \ldots, N_{i^{\prime}}\right\}} x_{i^{\prime} j^{\prime}} j T+E_{i^{\prime} k}
\end{array}\right) \\
-\frac{1}{a_{k}}
\end{array}\right) \delta_{i i^{\prime} k}^{+} \geq 0, k \in K, i, i^{\prime} \in I_{k}, i \neq i^{\prime} \\
\left(\begin{array}{l}
\left.\sum_{j \in\left\{0,1, \ldots, N_{i}\right\}} x_{i j} j T+E_{i k}\right) \\
\left(\begin{array}{l}
\sum_{j^{\prime} \in\left\{0,1, \ldots, N_{i^{\prime}}\right\}} x_{i^{\prime} j^{\prime}} j T+E_{i^{\prime} k}
\end{array}\right) \\
+\frac{1}{a_{k}}
\end{array}\right) \delta_{i i^{\prime} k}^{-} \leq 0, k \in K, i, i^{\prime} \in I_{k}, i \neq i^{\prime} \\
\delta_{i i^{\prime} k}^{+}+\delta_{i i^{\prime} k}^{-} \geq 1, k \in K, i, i^{\prime} \in I_{k}, i \neq i^{\prime} \\
x_{i} \in\{0,1\}, i \in I \\
d_{i j} \in\{0,1\}, i \in I, j \in\left\{0,1, \ldots, N_{i}\right\} \\
\delta_{i i^{\prime} k}^{+}, \delta_{i i^{\prime} k}^{-} \in\{0,1\}, k \in K, i, i^{\prime} \in I_{k}, i \neq i^{\prime}
\end{array}\right.
\end{aligned}
$$

Since the acceptance rate of the sector is usually between 13 flights per minute, under the operation requirements of TBO, the CTA of flight entering the sector usually needs to be accurate to seconds. In this model, assuming that the time is discretely operated by 1 second, and the maximum delay time of each flight is set to 2 hours, that is, 7,200 seconds, taking 10,000 flights within a day as an example, and only considering the scale of the decision variable, the search space of this model will be greater than $2^{72000000}$. Assuming that there are 400 sectors in total, each flight passes through 10 sectors on average, and there are about 200 flights in each sector on average, so there are about $8 \times 10^{3}$ inequations in Formula (6) and about $1.6 \times 10^{7}$ inequations in Formula (7)(8)(9) respectively. Therefore, the whole model has about $4.8 \times 10^{7}$ constraints. Because the nonlinear programming problem cannot obtain the optimal solution in polynomial time, 
for such a large scale 0-1 integer nonlinear programming problem, it will not be able to obtain the optimal solution in finite time, and put a very high demand on the memory of the computing machine.

\section{Description of Method}

Therefore, it is our main expectation to solve this problem to construct a reasonable model, design an efficient solution method and obtain an acceptable suboptimal solution in the face of actual operation requirements. Based on this, this article attempts to combine the heuristic framework with the precise solution, and integrates the idea of the sequential decision based on time window, greedy strategy, and optimal solution, to design a set of pre-tactical 4DT planning method based on the mixed optimization strategy (see Figure 2).

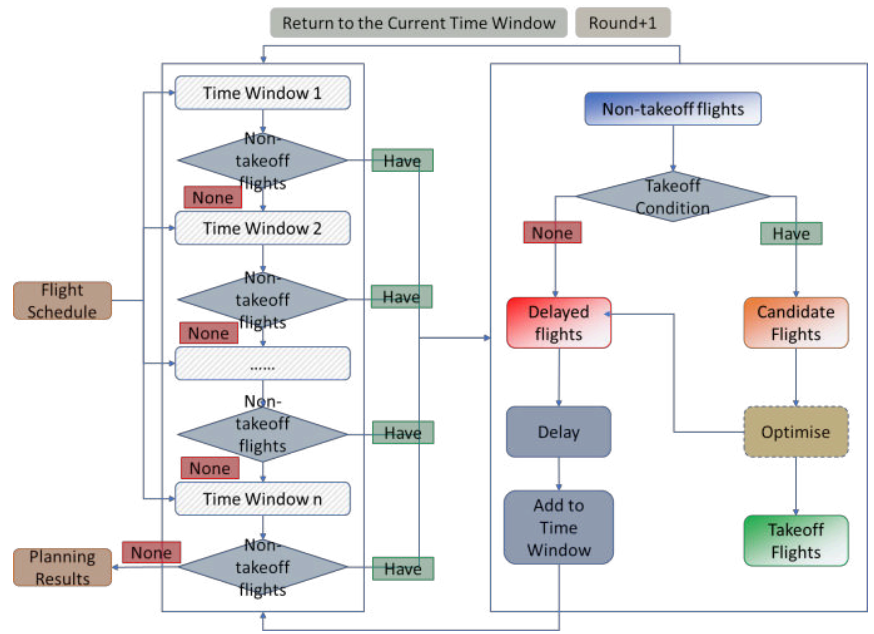

Figure 2. The Schematic diagram of pre-tactical 4DT planning method.

Firstly, the method divides all flights into time windows according to their departure time, and the length of the time window can be set according to the need. Starting from the earliest time window, the flight within the time window is judged one by one whether it has the take-off conditions. The so-called take-off condition refers to whether the flight if it takes off at the current planned time, is general conflict-free with the flight that has decided to take off. If so, it has the takeoff condition and vice versa. The flight with take-off condition within this time window is called a Candidate Flight, and the flight without take-off condition is called a Delayed Flight. Note that the candidate flights are not necessarily general conflict-free because the judgment of take-off conditions only depends on the flights decide to take off. Then, an optimization operation with the minimum number of delayed flights as the optimization goal is carried out for all the candidate flights in this time window (see the optimization model in paragraph E). Based on the optimization results, we decide which flights to take off and which flights to consider as delayed flights. Perform delay actions on all delayed flights. Delay operation refers to the delay of a delayed flight to the nearest time when it has the take-off condition. The above process is called an optimization round within a time window. At the end of the round, there are three possible outcomes for the flight that was in the time window at the start of the round: the flight decides to (1) take off, (2) delay and still in the current time window, and (3) delay but in a later time window. Therefore, after the end of a round, it is still necessary to check whether there are any flights in the current time window that have not taken off. If there are non-takeoff flights, the next round proceeds in the time window until all flights in this time window have decided to take off or delay to a later time window. The above process is then repeated for the next window until all flights have decided to take off.

In this method, the sequential decision based on the time window is reflected in that all flights make decisions according to the order of the time window and the decision of the flight in the later time window is affected by the flight in the earlier time window. Greedy strategy is reflected in the heuristic idea that the method is based on minimizing the number of delayed flights in each time window to reduce the total number of delayed flights. The optimal solution is reflected in each round within the time window, and the optimal model algorithm is used to solve the solution to get the minimum number of delayed flights in the round.

\section{E. Optimization Model}

To apply the general conflict-free constraint to any two candidate flights in the optimization model, we first need to construct the general conflict correlation matrix of flights which can be represented as:

$$
A=\left[\begin{array}{ccc}
\alpha_{11} & \cdots & \alpha_{1 j} \\
\vdots & \ddots & \vdots \\
\alpha_{i 1} & \cdots & \alpha_{i j}
\end{array}\right]
$$

where $a_{i j}$ is a binary parameter. 0 and 1 represent flight $i$ and flight $j$ is general conflict-free or not, respectively. Taking the minimum number of delayed flights as the optimization objective, a 0-1 integer linear programming model was established. The objective function is:

$$
\min Z=\sum_{i \in I_{\text {candidate }}}\left(1-x_{i}\right)
$$

where $x_{i}$ is the $0-1$ decision variable. 1 and 0 represent the flight $i$ is delayed or not, respectively. $I_{\text {candidate }}$ represents a set of candidate flights for the current round. Since this optimization model only needs to consider whether there is a general conflict between flight candidates, there is only one constraint condition:

$$
x_{i}+x_{j} \leq 2-\alpha_{i j}, i, j \in I_{\text {candidate }}, i \neq j
$$

Formula (14) means that if flight $i$ and flight $j$ are generical conflict-free, then $\alpha_{i j}$ is 0 , that is, the right side of the inequality is 2 , then flight $i$ and flight $j$ can choose to take off at the current scheduled takeoff time at the same time; if flight $i$ and flight $j$ are not generical conflict-free, then $\alpha_{i j}$ is 1 , that is, the right side of the inequality is 1 , then at most one of flight $i$ and flight $j$ can choose to take off at the 
currently scheduled takeoff time and the other have to delay in this round.

To sum up, the optimization model can be integrated as follows:

$$
\begin{aligned}
& \min Z=\sum_{i \in I_{\text {candidate }}}\left(1-x_{i}\right) \\
& \text { s.t. } \\
& \left\{\begin{array}{l}
x_{i}+x_{j} \leq 2-\alpha_{i j}, i, j \in I_{\text {candidate }}, i \neq j \\
x_{i} \in\{0,1\}, i \in I_{\text {candidate }}
\end{array}\right.
\end{aligned}
$$

If 10000 flights within a day are still taken as an example and the length of the time window is set as 20 minutes, there are about 139 flights in each time window on average. In each time window, the search space of the optimization model in each round on average is about $2^{139}$, and the constraint conditions are about $C_{139}^{2}=9591$. Although the 0-1 integer linear programming problem also cannot obtain the optimal solution in polynomial time, the scale of the problem is very small, and each calculation can be completed in millisecond time. Therefore, this method can support repeated calculation in a short time to realize sequential decision.

\section{F. Pseudocode} 1):

The pseudocode for this algorithm is as follows (Algorithm

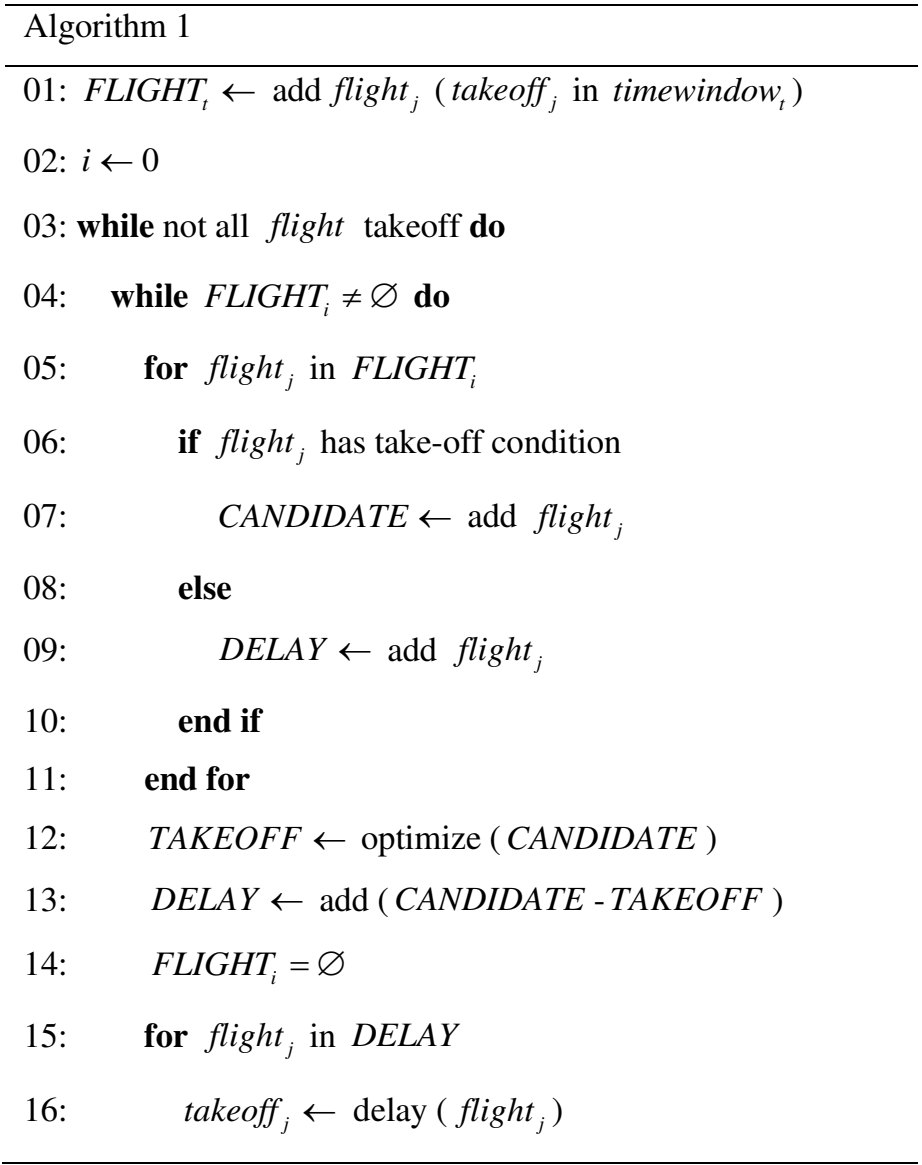

17: $\quad$ FLIGHT $_{t} \leftarrow$ add flight $_{j}\left(\right.$ takeoff $_{j}$ in timewindow $\left._{t}\right)$

18: $\quad$ end for

19: end while

20: $\quad i \leftarrow i+1$

\section{1: end while}

\section{G. Advantages}

Compared with the optimization model based on time discretization, the proposed method has the following advantages:

a) Based on the continuous-time model, this method can get the accurate minimum delay time in the delay time calculation, avoiding the loss of precision caused by time discretization, thus increasing the flight delay time.

b) In this method, the large-scale optimization problem is divided into parts and transformed into a heuristic method based on sequential decision making. The problem size of each sub-problem is greatly reduced and the solving speed is greatly accelerated.

c) This method is based on the sequential decision of greedy strategy, and can better support the dynamic updating of planning results. Even if the results of previous planning are not implemented accurately in real-time due to other factors, the method can quickly update the planning results based on the near real-time situation of previous flights, which also makes the method potentially compatible with sector dynamic capacity configuration.

\section{NUMERICAL ANALYSIS}

To verify the relevant performance of the proposed method, we used the real flight schedule data of the Network Strategic Modelling Tool (NEST) developed by EUROCONTROL over the airspace of Spain and France on February 14, 2017, for simulation. A total of 376 sectors and 8,153 flights are covered (see Figure 3).

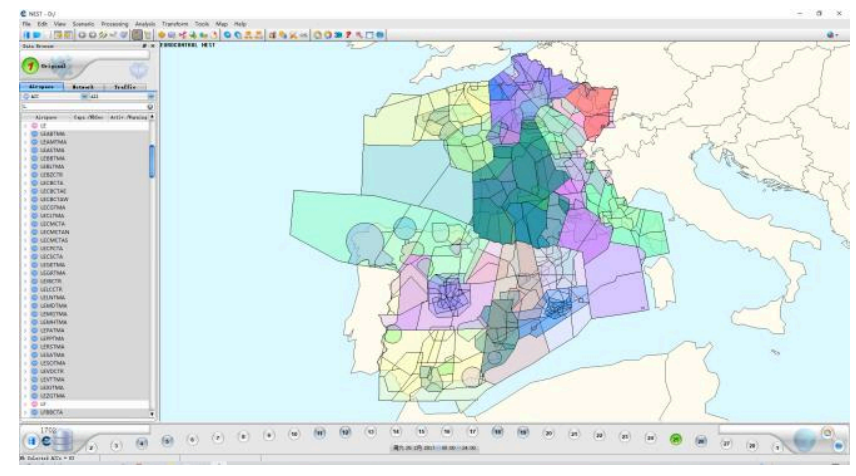

Figure 3. French and Spanish sectors in NEST.

The experiment in this article is based on Intel(R) Core (TM) I7-10510U CPU @ $1.80 \mathrm{GHz} 2.30 \mathrm{GHz}$ and 64-bit 
Windows 10 processing system, using Python 3.8 programming language.

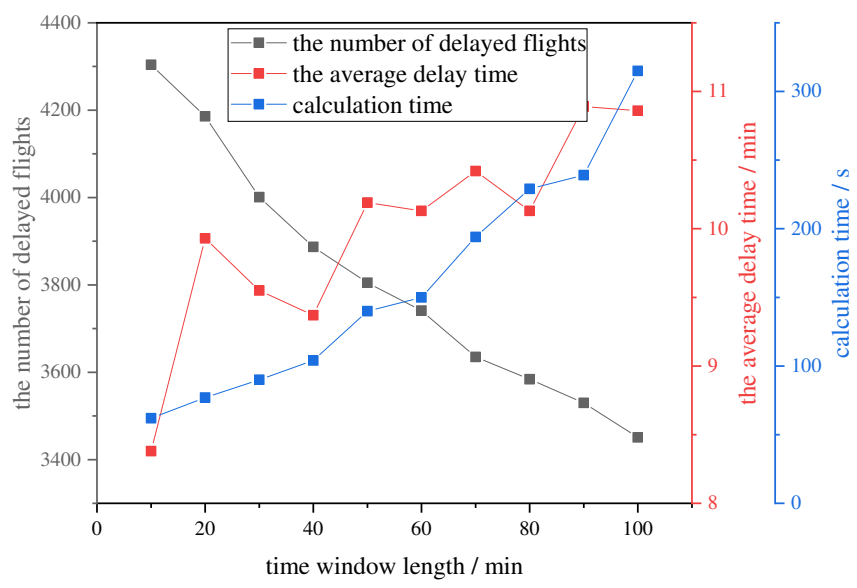

Figure 4. Simulation results of the hybrid optimization strategy method.

We test the performance parameters of the method in this article under different time window lengths, including the number of delayed flights, average delay time and calculation time. Among them, the number of flight delays refers to the number of flights not taking off on time according to the flight schedule; Average delay time refers to the quotient of the total delay time of all flights and the total number of flights; Calculation time refers to the time taken by the algorithm to complete all the calculations. As can be seen from Figure 4:

a) As the length of the time window increases, the number of flight delays decreases continuously, because when the length of the time window approaches 0 , it is equivalent to adopting a first-come-first-served strategy. However, the longer the time window is, the greater the benefits brought by the optimization model based on the optimization objective, namely the minimum number of flight delays. This trend indicates that the optimization model of the algorithm is effective to minimize the number of flight delays, and the whole algorithm is effective in reducing the number of flight delays in the pre-tactical stage.

b) As the length of the time window expands, the average delay time fluctuates and increases, which is in line with common sense, because when the length of the time window approaches infinitely to the entire optimization time range, it is equivalent to the global optimization of the problem. Driven by the optimization goal of minimizing the number of flight delays, flights will take off on time as much as possible, resulting in less flexibility of flight adjustment and thus an increase in the average delay time.

c) As the length of the time window expands, the calculation time shows an accelerated upward trend, because the longer the time window is, the more flights are available in each time window. Also, because the calculation time of the optimization model of the algorithm increases exponentially with the size of the problem, the calculation time increases rapidly. d) From the absolute, time window length is 100 minutes, for example, compared with the first-come-first-served strategy, flight punctuality increased by about $20 \%$, the average delay time and control under the current actual operation level of delay (2016 Europe average delay time was 11.3 minutes per flight [10]), and computation time is kept in 5 minutes or so. It can meet the demand of the dynamic update in practical operation.

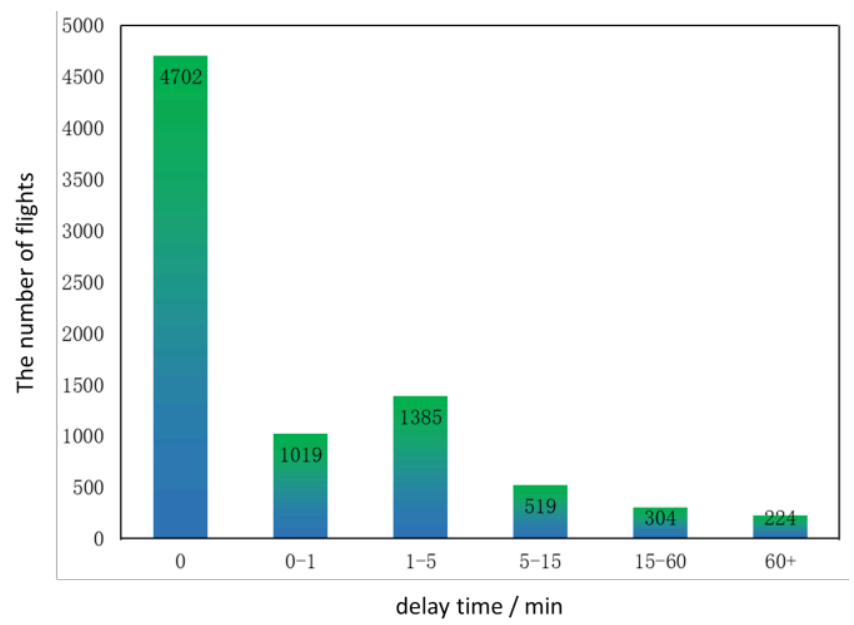

Figure 5. The statistics flight delay time.

Figure 5 shows the optimization results with a time window length of 100 minutes. It can be found that most delayed flights are delayed within 15 minutes. Flights delayed by more than 15 minutes accounted for only 6.5 per cent of all flights delayed and 2.8 per cent of all flights.

At present, there are few reports on the research of DCB considering general conflict-free in the TBO environment, and the method proposed in this article is oriented to the DCB problem in the pre-tactical stage of practical operation. Therefore, we compare this method with CASA, which is used in the practical operation of European ATM to deal with the DCB problem. Because CASA is not designed based on the TBO environment, it cannot guarantee that the optimized flight trajectory is completely general conflict-free, and can only guarantee that flights within the hotspot strictly conform to the sector acceptance limit. The hotspot in CASA refers to that the number of flights entering within a certain time window of the sector is greater than the capacity of the sector within the length of the time window. If the number of flights entering within a time window of each sector is greater than the capacity of the sector within the length of the time window, it is denoted as one hotspot. Besides, since CASA often adopts the method of manually increasing the sector capacity parameters in practical operation to make the results more consistent with the practical operation requirements, we uniformly increased the capacity of all sectors under CASA operation by $15 \%$ in the comparative experiment. Therefore, this comparison is not equal. For the general conflict-free constraint of the trajectory, CASA adopts a more relaxed constraint, while the method proposed in this article adopts a completely strict constraint. Therefore, we minimize the length of the time window in CASA as much as possible to generate more hotspots, to make 
its optimization constraints as close as possible to the method in this article. This comparison is to provide readers with an objective reference as much as possible.
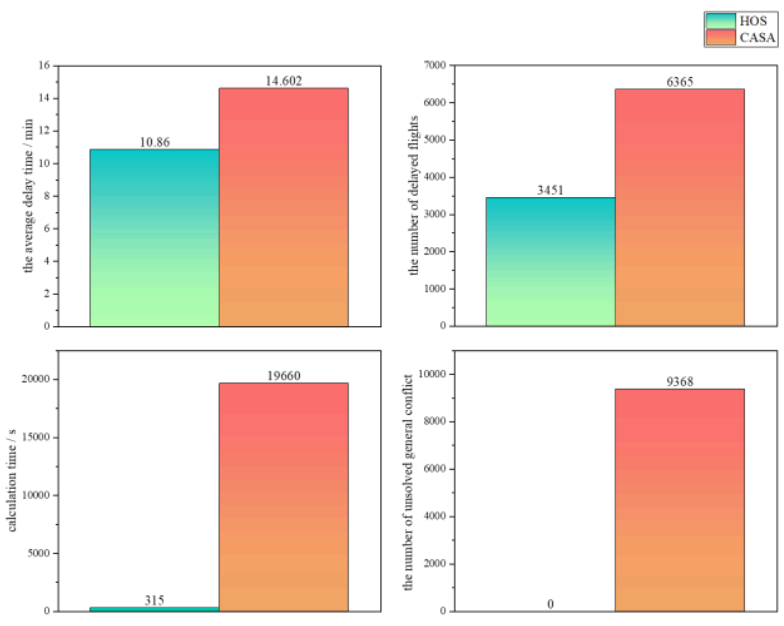

Figure 6. The result of the comparison between HOS and CASA.

Figure 6 shows the results of the comparative experiment, where the time window length of the method based on the HOS in this article is 100 minutes, and the time window length of CASA is 1 minute. In CASA, there are a total of 1246 hotspots before optimization. After iterating for 300 rounds, the hotspots have not been solved completely, and there are still 162 hot sectors that accounted for about $13 \%$ of the initial hotspots. In the practical operation, however, CASA can have a smaller proportion of the hotspots that have not been solved (usually $15 \%$ ), so it is meet the requirements of the practical operation. The data shown in Figure 6 is the result of CASA after completing 300-round iterations. It can be found that:

a) In terms of average delay time, compared with CASA, the proposed method reduces by $25.6 \%$, indicating that the proposed method has a significant effect on reducing the delay time.

b) In terms of the number of delayed flights, compared with CASA, the method in this article reduces by $45.8 \%$, indicating that the proposed method has a significant effect on improving the on-time rate.

c) In terms of calculation time, the time spent in 300 rounds of CASA iteration is 19,660 seconds, that is, 5.46 hours, which can only support d-1 capacity allocation. However, the method in this article only takes 315 seconds, which is only $1.6 \%$ of CASA and has great potential to support dynamic updating of planning results and to be compatible with dynamic sector capacity allocation.

d) In terms of conflict resolution of G4DT, CASA due to the relative relaxation constraint, there is no guarantee that all flights are general conflict-free, so in the 300-round iteration after there are still 9368 unresolved general conflicts (in the same sector within each has two flights do not conform to the sector to accept rate constraint is recorded as a general conflict and each sector are calculated respectively). However, the method in this article achieves the genera conflict-free of all flights, which meets the operational requirements of TBO.

\section{CONCLUSION AND PROSPECT}

In this article, for the future TBO operation environment, a set of pre-tactical-and-tactical integrated 4DT planning framework is proposed. For the DCB problem, a G4DT planning method based on an HOS is designed. Experiments show that this method can effectively reduce the delay based on completely solving the general conflict. Thanks to its highspeed computing performance, the results can be updated dynamically according to real-time information. In this article, the DCB problem is solved only by GDP. In the future, rerouting can be further added to the G4DT planning. Besides, relying on the advantages of the TBO operating environment, the flight time in each sector can be dynamically adjusted according to the aircraft performance, so that the trajectory optimization has greater flexibility, thus further reducing delays.

\section{ACKNOWLEDGMENT}

The experimental environment and data for this article are provided by the ATM lab of SATM, Cranfield University. This study is funded by China Scholarship Council, National Natural Science Foundation of China (No.61903187), Natural Science Foundation of Jiangsu Province (BK20190414), Postgraduate Research Innovation Program of Jiangsu (KYCX20_0213) and Interdisciplinary Innovation Foundation for Postgraduates of NUAA (KXKCXJJ202001).

\section{REFERENCES}

[1] ICAO. "Doc 9854: Global Air Traffic Management Operational Concept International Civil Aviation Organization." 2005.

[2] CHOROBA, P., \& van der HOORN, L. "Towards a more harmonised and wider use of short-term atfcm measures (STAM). " $30^{\text {th }}$ Congress of the International Council of the Aeronaurical Sciences. DCC, Daejeon, Korea. September 25-30, 2016.

[3] Ruiz, S., Kadour, H., \& Choroba, P. "An Innovative Safety-neutral Slot Overloading Technique to Improve Airspace Capacity Utilisation. " $9^{\text {th }}$ SESAR Innovation Days. December 2-5, 2019.

[4] Rebollo, J. J., \& Cruz, A. "Hybrid demand and capacity balance model for the future air traffic management concept of operations. " Eighth USA/Europe Air Traffic Management Research and Development Seminar. 2009.

[5] Kravaris, T., Vouros, G. A., Spatharis, C., Blekas, K., Chalkiadakis, G., \& Garcia, J. M. C. "Learning policies for resolving demand-capacity imbalances during pre-tactical air traffic management. "German Conference on Multiagent System Technologies. Springer, Cham, 2017.

[6] Shetty, K., Gulding, J., Koelman, H., Celiktin, M., \& Koelle, R.. "Comparison of ATFM practices and performance in the US and Europe. "2017 Integrated Communications, Navigation and Surveillance Conference (ICNS). IEEE, 2017.

[7] Che, J. http://att.caacnews.com.cn/gcsy/201912/t20191227_20770.html

[8] Gardi, A. G. A Novel Air Traffic Management Decision Support System Diss. RMIT University, 2016.

[9] Gatsinzi, D., Saez Nieto, F. J., \& Madani, I. "Development of a new method for ATFCM based on trajectory-based operations." Proceedings of the Institution of Mechanical Engineers, Part G: journal of aerospace engineering 233.1 (2019): 261-284.

[10] Xu, Y., Dalmau, R., Melgosa, M., Montlaur, A., \& Prats, X. "A framework for collaborative air traffic flow management minimizing costs for airspace users: Enabling trajectory options and flexible pre- 
tactical delay management." Transportation Research Part B: Methodological 134 (2020): 229-255.

[11] Chen, Y., Yang, L., Zhang, H., Zhao, Z., \& Hu, M."Real-Time Autonomous Trajectory Conflict Detection and Resolution in Restricted Airspace." 2020 Integrated Communications Navigation and Surveillance Conference (ICNS). IEEE, 2020.

[12] Koyuncu, E., \& Başpınar, B. "Demand and Capacity Balancing Through Probabilistic Queuing Theory and Ground Holding Program for European Air Transportation Network." Anadolu Üniversitesi Bilim Ve Teknoloji Dergisi A-Uygulamalı Bilimler ve Mühendislik 18.2 (2017): 360-374. 
$2021-11-15$

\section{A 4D-trajectory planning method based on hybrid optimization strategy for demand and capacity balancing}

Chen, Yutong

IEEE

Chen Y, Xu Y, Hu M, et al., (2021) A 4D-trajectory planning method based on hybrid optimization strategy for demand and capacity balancing. In: 2021 AIAA/IEEE 40th Digital Avionics Systems Conference (DASC), 3-7 October 2021, San Antonio, USA http://doi.org/10.1109/DASC52595.2021.9594296

Downloaded from Cranfield Library Services E-Repository 